\title{
Acute Simultaneous Renal and Ovarian Vein Thrombosis Mimick- ing Renal Colic and Associated with Factor V Leiden: Case Report and Review of Literature
}

\author{
Chahid Farah', Sabine Imad', Rachelle Abboud ${ }^{2}$, Fady Nader', Rahil Zeaiter ${ }^{2}$, Boutros \\ Youssef ${ }^{2}$, Karen Nicolas ${ }^{3}$, Mireille Rizkallah ${ }^{3}$, Alain Khalaf ${ }^{4}$ and Tony El Murr ${ }^{*}$ \\ ${ }^{1}$ Department of Surgery, Faculty of Medical Sciences, Lebanese University, Beirut, Lebanon \\ ${ }^{2}$ Department of Internal Medicine Diseases, Faculty of Medical Sciences, Lebanese University, Beirut, Lebanon \\ ${ }^{3}$ Division of Radiology, MEIH-UH Affiliated with the Faculty of Medical Sciences, Lebanese University, Bsalim, Lebanon \\ ${ }^{4}$ Head of Urology Division, MEIH-UH Affiliated with the Faculty of Medical Sciences, Lebanese University, Lebanon \\ ${ }^{5}$ Division of Internal Medicine Diseases, Head of Medicine Department, Faculty of Medical Sciences, Lebanese University, \\ Lebanon
}

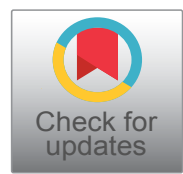

*Corresponding author: Tony EI Murr, MD, Division of Internal Medicine Diseases, Head of Medicine Department, MEIHUH Affiliated with the Faculty of Medical Sciences, Lebanese University, Bsalim, Lebanon, Tel: 961-3347473

\begin{abstract}
Severe flank pain is a frequent complaint at the emergency department (ED). It is usually associated to other clinical symptoms like fever, dysuria, vomiting, diarrhea or radiation to the groin. Hereby, it may raise a lot of probable differential diagnosis that should be ruled out first depending on laboratory tests and imaging results. However, when flank pain is isolated and radiating to the groin without evidence of urolithiasis on abdominal CT scan, more rare diagnosis should be suggested such as renal vessels thrombosis with or without other vessel thrombosis in the pelvis. Thereafter, thrombophilic studies should be performed to elucidate the underlying etiology. We present the case of a 44-yearold lady having simultaneously acute left renal and ovarian veins thrombosis presenting to ED for severe isolated left flank pain radiating to the left groin and heterozygous factor $\checkmark$ Leiden on thrombophilic studies. We would like to stress on the importance of having a high index of suspicion of such diagnosis at ED based on medical history and primary investigations because a good management may result in salvage of organs by minimally invasive techniques, an early effective treatment and convenient anticoagulation in the future to prevent further complications.
\end{abstract}

\section{Keywords}

Flank pain, Thrombosis, Ureterolithiasis, Anticoagulation

\section{Introduction}

Hypercoagulable state known as thrombophilia is the main factor predisposing to thromboembolic events. It was described by Rudolf Virchow in the nineteenth century as a constituent factor of a triad known to be the cause of any thromboembolic events and including: Stasis, endothelial injury, and hypercoagulability state. It may be inherited or acquired and should be suspected in patients with venous or arterial thrombosis of young age, unusual locations, recurrent thrombosis, pregnancy loss or positive family history.

We discuss the case of a young previously healthy lady that was presented for intractable left flank pain without any other associated symptoms and was found to have left renal and ovarian venous thrombosis probably due to a heterozygous mutation in factor $\mathrm{V}$.

\section{Case Presentation}

A 44-year-old female patient, bank officer, presented for the first time to our university hospital ED for severe left flank pain lasting since few hours. The pain was achy and dull, radiating to the left groin, increasing dramatically despite common traditional analgesics like

Citation: Farah C, Imad S, Abboud R, Nader F, Zeaiter R, et al. (2020) Acute Simultaneous Renal and Ovarian Vein Thrombosis Mimicking Renal Colic and Associated with Factor V Leiden: Case Report and Review of Literature. Clin Med Rev Case Rep 7:297. doi.org/10.23937/2378-3656/1410297 Accepted: February 12, 2020: Published: February 14, 2020

Copyright: (c) 2020 Farah C, et al. This is an open-access article distributed under the terms of the Creative Commons Attribution License, which permits unrestricted use, distribution, and reproduction in any medium, provided the original author and source are credited. 

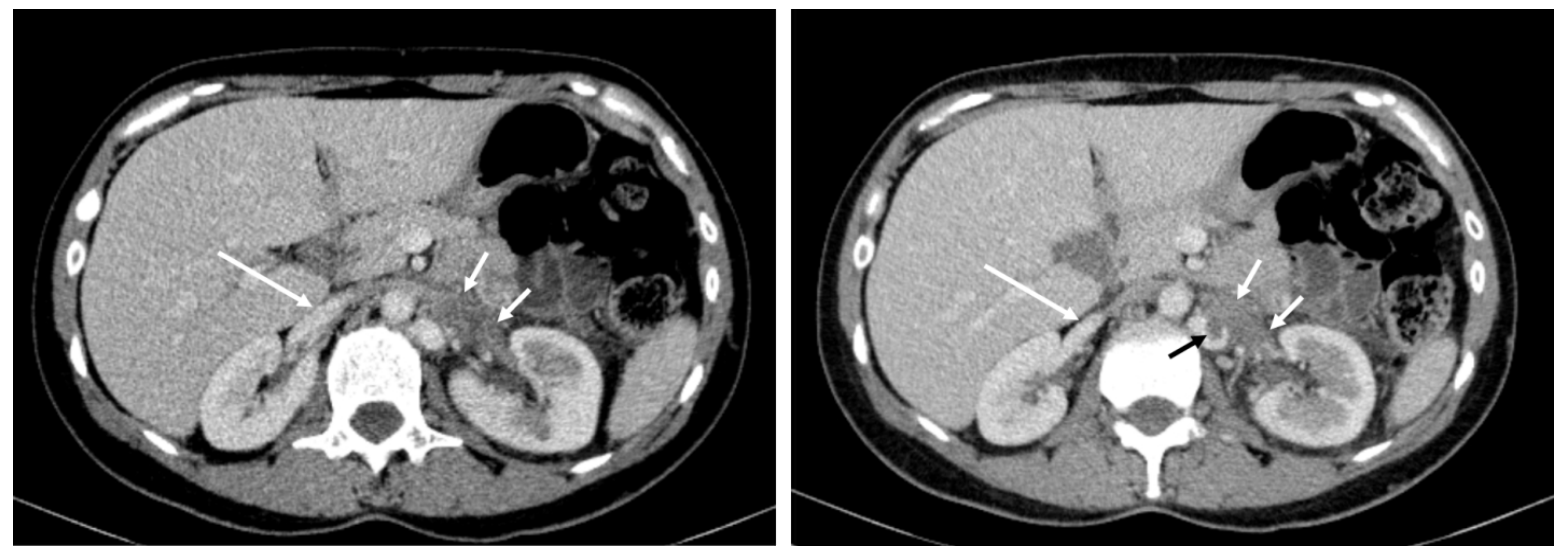

Figure 1: Right renal vein normally opacified after contrast injection (long arrow); Left renal vein (short white arrows) enlarged, hypodense, not perfused in favor of acute thrombosis. Continuous intravenous hypodense thrombus (black arrow) in the upper part of the ovarian vein (black arrow), the lumen of the ovarian vein is enlarged due to the obstruction of the renal vein above.

nonsteroidal anti-inflammatory and spasmolytic drugs, and associated only with nausea. She denies another gastrointestinal or urinary symptoms. She has had no fever or chills nor paresthesia or lower limbs weakness. She is non-smoker, drinks alcohol occasionally, takes no chronic medication, except acetylsalicylic acid, and has no known allergies. She is not taking any oral contraceptive hormones since more than 5 years.

On admission, her blood pressure (BP) was 140/85 $\mathrm{mmHg}$, body temperature of $38{ }^{\circ} \mathrm{C}$, respiratory rate 22 , heart rate $95 \mathrm{bpm}$, and oxygen saturation $98 \%$ on room air. Her physical examination revealed a conscious, well-nourished, non obese $\left(B M I=27 \mathrm{~kg} / \mathrm{m}^{2}\right)$ patient with a normally-colored skin and conjunctiva. She has left costovertebral angle (CVA) tenderness and left lower quadrant abdominal pain on deep palpation. Her cardio-pulmonary auscultation is normal and she has no weight loss, no buccal ulcers, no malar rash or arthralgia, no lower limb edema, and no palpable peripheral lymph nodes.

In her past medical history, she reported post-partum left lower limb deep venous thrombosis (DVT) three years ago that was treated with low molecular weight heparin (LMWH) for only 3 months and was followed one year later by a mild spontaneous superficial right arm vein thrombosis. She was treated by local heparinoid cream (Hirudoid) and put on low dose acetylsalicylic acid (100 mg per day) since that time.

At ED, her blood tests showed slightly elevated peripheral white blood cells (WBC $=11.5 \mathrm{G} /$ liter; $68 \%$ neutrophils and $22 \%$ lymphocytes), hemoglobin of $13.6 \mathrm{~g} /$ $\mathrm{dL}$, and platelet count of $362000 / \mu \mathrm{L}$. Serum glucose, C-reactive protein (CRP), serum creatinine, serum liver function tests, serum albumin and serum lipase level were all within normal range. Serum cholesterol, serum complement and antinuclear antibodies (ANAs) were also normal. Urinalysis showed numerous red blood cells and few leukocyte count (8-10/hpf). No crystals, proteins or casts were found in the urine. Urine culture was sterile and chest $\mathrm{X}$-ray was normal.

Non contrast-enhanced abdominal CT showed first an ill-defined calcification that was misdiagnosed with left ureterolithiasis but the diagnosis was adjusted one hour later by injecting intravenous contrast. The patient was found to have mixed left renal and left ovarian vein thrombosis with a clear-cut absence of any obstruction on the urinary tract. Left kidney was slightly enlarged without evidence of any tumor or infarction. There was no ascites or enlarged intra-abdominal lymph nodes (Figure 1).

Treatment with LMWH and analgesic drugs was started immediately, and the patient was constantly improving in the next few days. She left hospital on the fourth day following her admission. Thrombophilic studies performed six weeks later revealed factor $\checkmark$ heterozygous mutation (Factor $V$ Leiden) and the patient was put on long life treatment with new oral anticoagulation agent rivaroxaban $20 \mathrm{mg}$ per day.

\section{Discussion}

Renal vein thrombosis (RVT) is a relatively rare clinical entity. It is commonly associated with nephrotic syndrome or direct invasion by renal cell cancer. Other less common causes include hypercoagulable states, extrinsic compression by tumors, infections, trauma, renal transplantation, Behcet syndrome or antiphospholipid antibody syndrome [1,2]. Almost two thirds of patients have bilateral renal vein involvement. In cases of unilateral thrombosis, the left renal vein is affected more commonly than the right one [3]. The severe passive congestion causing the kidney to become engorged, leads to degeneration of nephrons and causing symptoms of flank pain, hematuria and decreased urine output.

The presentation of renal vein thrombosis is usually variable and vary by the rapidity of the venous occlusion; patients may be asymptomatic or having 
symptoms related only to the underlying etiology. Acute renal vein thrombosis usually presents with symptoms of renal infarction, including flank pain, flank tenderness, rapid deterioration of renal function and worsening proteinuria, micro or macroscopic hematuria. It may also present with pulmonary emboli [4]. In normal Western populations, heterozygosity for the factor $\mathrm{V}$ Leiden mutation is present in $2-5 \%$, whereas in patients with venous thrombosis and a family history of thrombotic disease this figure may reach $50-60 \%$. The presence of the mutation markedly increases the risk for renal vein thrombosis, particularly in neonates [5].

Ovarian vein thrombosis is a rare complication which arises classically in the post-partum [6]. This condition is classically a puerperal process, but may also be caused by endometritis, pelvic inflammatory disease, malignancy, thrombophilia, inflammatory bowel disease, and post-operatively [7]. This condition involves the right vein more commonly than the left. Ovarian vein thrombosis often has a vague and variable presentation, and a high index of suspicion is required to make the diagnosis [8]. Some patients will have nonspecific symptoms, including malaise, vague diffuse abdominal pain, or dyspnea; others will present with fever and lower quadrant pain [9].

Our patient has had most probably an acute rather than chronic venous thrombosis since the pain had rapid onset and was only associated to a recent microscopic hematuria without evidence of a chronic urinary or gynecologic manifestations. The renal vein thrombosis was on the left side like most of the cases described in the literature unlike the ovarian thrombosis. Our patient has both thrombosis on the left side which is concordant with many reports in the literature showing that left-sided renal vein thrombosis can lead to left gonadal vein thrombosis with pelvic congestion in females and painful swelling of the left testis in males [10]. The only positive factor that was indicating the possibility of thrombosis in this young healthy lady, was the presence of previous DVT in post-partum. Otherwise, ureterolithiasis has been evoked as the primary diagnosis owing to the type and localization of pain at ED.

Factor $\mathrm{V}$ Leiden thrombophilia is characterized by a poor anticoagulant response to activated protein $C$ (APC) and an increased risk for venous thromboembolism (VTE) [11]. It also increases the VTE risk in "unusual locations" not routinely seen.

Heterozygous Factor $V$ Leiden carriers have 5 to 10 times increased risk of thrombosis in comparison to the normal population while homozygous carriers have 50 to 100 times this risk. Other thrombophilic conditions such as the use of oral contraceptives are usually present in case of thrombosis due to heterozygous mutation.
Another interesting point to discuss in our clinical case is the causal relationship between the venous thrombosis and the presence of factor $\mathrm{V}$ Leiden. The imputability is hard to accept since this heterozygous mutation is usually asymptomatic and may be present in around $3 \%$ of the normal population. Moreover, it is rarely the only causative factor in unprovoked DVT and the previous lower limb DVT of our patient has occurred in the post-partum state. Nevertheless, no other medical or surgical predisposing situation was present during this episode of thrombosis and blood tests and imaging studies did not show any evidence of nephrotic syndrome, renal cancer or other abdominal tumors, abdominal trauma or infection, connective tissue disease or antiphospholipid syndrome. Therefore, heterozygous factor $\mathrm{V}$ Leiden remained the only etiology to admit.

Venous thrombosis associated to factor $\mathrm{V}$ Leiden should be diagnosed and treated by long term anticoagulation (ie, heparin, warfarin). Duration of treatment is defined on case by case and depends on whether the mutation is heterozygous or homozygous, recurrent or first episode, provoked or unprovoked, associated or not to pulmonary emboli. In general, recurrent unprovoked venous thrombosis due to homozygous mutation should be treated for life [12]. Reduction in proteinuria, by the use of angiotensin-converting enzyme inhibitors (ACEIs) or angiotensin-receptor blockers (ARBs) is essential in the treatment of renal vein thrombosis (RVT) in patients who are nephrotic.

The role for thrombolysis in the treatment renal venous thrombosis is unclear yet since no data are available comparing thrombolytic therapy with anticoagulation [13]. When anticoagulation is contraindicated, a vena cava filter must be placed at a suprarenal level to reduce the risk of pulmonary emboli. Surgical treatment is no more indicated unless there is underlying renal cell carcinoma [14].

\section{Conclusion}

Renal vein thrombosis is a rare complication of many hereditary or acquired hypercoagulable state such as heterozygous mutation of factor $V$. In its acute presentation, it may be confounded with renal colic crisis especially when associated with ipsilateral gonadal vein thrombosis. Its occurrence is highly suspected in the presence of acute flank pain, resistant to traditional analgesics, the absence of ureterolithiasis on CT scan and a medical or familial history of hypercoagulable state. Prompt diagnosis and convenient etiologic management are essential to avoid further invasive investigations and unexpected complications.

\section{References}

1. Barbour SJ, Greenwald A, Djurdjev O, Levin A, Hladunewich MA, et al. (2012) Disease-specific risk of venous 
thromboembolic events is increased in idiopathic glomerulonephritis. Kidney Int 81: 190-195.

2. De Azevedo FVA, Maia DG, de Carvalho JF, Rodrigues CEM (2018) Renal involvement in antiphospholipid syndrome. Rheumatol Int 38: 1777-1789.

3. Asghar M, Ahmed K, Shah S, Siddique M, Dasgupta P, et al. (2007) Renal Vein Thrombosis. European Journal of Vascular and Endovascular Surgery 34: 217-223.

4. Decoster T, Schwagten V, Hendriks J, Beaucourt L (2009) Renal colic as the first symptom of acute renal vein thrombosis, resulting in the diagnosis of nephrotic syndrome. Eur J Emerg Med 16: 170-171.

5. Wüthrich, R (2001) Factor $V$ Leiden mutation: Potential thrombogenic role in renal vein, dialysis graft and transplant vascular thrombosis. Curr Opin Nephrol Hypertens 10: 409414.

6. Salomon O, Dulitzky M, Apter S (2010) New observations in postpartum ovarian vein thrombosis: Experience of single center. Blood Coagul Fibrinolysis 21: 16-19.

7. Ginsberg JS, Bates SM (2003) Management of venous thromboembolism during pregnancy. J Thromb Haemost 1: 1435-1442.
8. Jenayah AA, Saoudi S, Boudaya F, Bouriel I, Sfar E, et al. (2015) Ovarian vein thrombosis. The Pan African Medical Journal 21: 251.

9. Martinelli I (2006) Thromboembolism in women. Semin Thromb Hemost 32: 709-715.

10. Mazhar HR, Aeddula NR (2019) Renal Vein Thrombosis. StatPearls, Treasure Island (FL).

11. Kujovich JL (1999) Factor V Leiden Thrombophilia. In: Adam MP, Ardinger HH, Pagon RA, Wallace SE, Bean LJH, et al. GeneReviews. University of Washington, Seattle (WA), 1993-2019.

12. Kearon C, Akl EA, Ornelas J, Blaivas A, Jimenez D, et al. (2016) Antithrombotic therapy for VTE disease: CHEST guideline and expert panel report. Chest 149: 315-352.

13. McCarthy E, Mahony NO, Guiney M, Ryan JM (2011) Successful catheter directed thrombolysis of IVC and renal vein occlusive thrombus. Ir Med J 104: 311-312.

14. Jaako Dardashti V, Békássy ZD, Ljung R, Gelberg J, Wingren $P$, et al. (2009) Successful thrombolysis of neonatal bilateral renal vein thrombosis originating in the IVC. Pediatr Nephrol 24: 2069-2071. 